\title{
The In-orbit Performance of SEVIRI From Observations of Mercury and Venus
}

This paper was downloaded from TechRxiv (https://www.techrxiv.org).

\section{LICENSE}

CC BY 4.0

SUBMISSION DATE / POSTED DATE

$17-11-2021 / 29-11-2021$

\section{CITATION}

Burgdorf, Martin; Buehler, Stefan; John, Viju; Müller, Thomas (2021): The In-orbit Performance of SEVIRI From Observations of Mercury and Venus. TechRxiv. Preprint. https://doi.org/10.36227/techrxiv.17031872.v1

DOI

10.36227/techrxiv.17031872.v1 


\title{
The In-orbit Performance of SEVIRI From Observations of Mercury and Venus
}

\author{
Martin J. Burgdorf, Stefan A. Buehler, Viju O. John, Thomas G. Müller
}

\begin{abstract}
We investigated various aspects of the in-orbit performance of SEVIRI on Meteosat-10 (launch: 05 Jul 2012) and -11 (launch: 15 Jul 2015) with images, where Mercury or Venus appeared in a corner. These objects are of similar or smaller size than the instantaneous field of view, and therefore they are well suited for checks of geometric requirements. From comparing the position of Venus in different channels we conclude that the North-South distance between the two focal planes is shorter than the nominal value by $0.66 \pm 0.14 \mathbf{~ k m}$ at SSP (Sub-Satellite Point) with Meteosat-10 and longer by $1.44 \pm 0.16 \mathbf{~ k m}$ at SSP with Meteosat-11. The tilt of the detector array against the equator is less than $0.0037^{\circ}$ for SEVIRI on Metosat-10. The sampling with narrow channels is $3.0016 \mathrm{~km}$, with a one-sigma uncertainty of $30 \mathrm{~cm}$ at sub-satellite point. The tests we carried out to check the geometric performance of the instrument confirmed that SEVIRI is compliant with the requirements. The Point Spread Function as determined from the image of a planet agrees well with the expectations based on its combination with the finite impulse response. Finally we determined the stability of the calibration coefficients from the counts obtained on the planetary targets and found the reproducibility of the measurements of planetary fluxes similar to those of vicarious calibration targets. Hence planets are a promising alternative to established methods of in-flight characterisation and validation of imagers.
\end{abstract}

Index Terms-Image quality, infrared imaging, Mercury, Venus.

\section{INTRODUCTION}

$\mathbf{S}$ EVIRI (Spinning Enhanced Visible Infra-Red Imager) performs multi-purpose imagery, tracking among other things water vapour features. It has eleven narrow-bandwidth channels and is the primary instrument on Meteosat- 8 to Meteosat-11, also known as MSG-1, -2, -3, and -4 (Meteosat Second Generation), four satellites in geostationary orbit ${ }^{1}$, see Figure 1. The SEVIRI design was driven by stringent requirements on geometric performance [1], i. e. tight constraints on the positional variations of a pixel relative to the previous image or other pixels.

The Level 1.0 geometric image quality has been verified during the commissioning phase and found to meet the requirements [2], but some anomalies appeared over the lifetime of the satellites. The rectified images from the Rapid Scanning Service of Meteosat-8, for example, became

M. Burgdorf and S. A. Buehler are with the Department of Earth Sciences, Universität Hamburg, 20146 Hamburg, Germany, e-mail: martin.burgdorf@uni-hamburg.de.

V. John is with EUMETSAT.

T. Müller is with Max-Planck-Institut für extraterrestrische Physik.

Manuscript received September 30

${ }^{1}$ https: / / space.oscar.wmo.int/satelliteprogrammes/ view/meteosat_2nd_generation

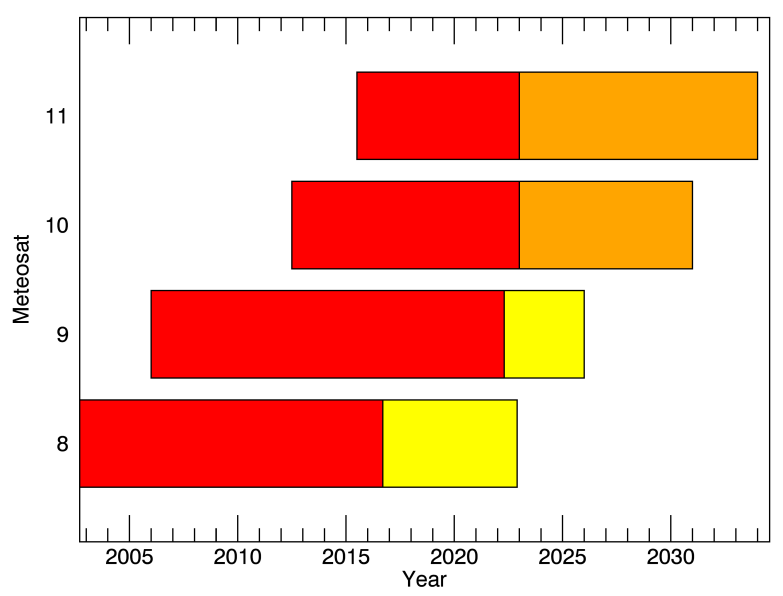

Fig. 1. Meteosat Second Generation - programme lifetime. In yellow: (expected) Indian Ocean data coverage, in orange: (expected) period until end of life.

increasingly tilted. What is more, very sudden absolute spacecraft movements of Meteosat- 8 and -9 caused jumps equivalent to two to five pixels in the SEVIRI images - this effect needed to be compensated via image processing [3]. But even the latest satellites are not free from problems, as demonstrated by Nain \& Mueller, who have recently found an inter channel residual registration error of $0.9 \mathrm{~km}$ in Level 1.0 images of Meteosat-11 [4]. Although this value is still within the limits set by the requirements, they recommended updating the pointing table for in-flight measurements. As there was no such work in the past, the question arises, whether a similar inter-channel residual registration error was present in previous satellites as well.

The geometric quality of the further processed SEVIRI Level 1.5 products is routinely assessed by the European Organisation for the Exploitation of Meteorological Satellites (EUMETSAT) in terms of absolute geolocation and interband registration based on a landmark detection approach [5]. A fundamental problem with checking the geometric performance of an infrared imager in flight is, however, that landmarks can only be found in window channels. Therefore measurements of the co-registration of sounding channels suffer from the fact that the radiation is received from different heights in the atmosphere. As the predominant wind patterns vary with height, matching images of the Earth from different channels is inevitably prone to systematic errors. By 
contrast an isolated point source in space is perfectly suited to investigate the geometrical optics, and it allows to monitor quality indicators without complicated matching methods and yet with higher accuracy than is possible with images of Earth. This is because planets appear in all channels at well-defined positions spanning the full size of the image.

With this idea in mind we decided to use images of SEVIRI with a planet in a corner for verifying the compliance with optical requirements while paying special attention to the instrumental anomalies detected in the past. Besides that, we investigated whether these images can also be helpful for checking the radiometric performance, in particular of the solar channels, where usually a vicarious calibration is used. Its accuracy is of the order 5\% [6], and the calibration coefficients are only updated every 45 days [2]. EUMETSAT performs therefore an additional drift monitoring, for which it employs the Moon as calibration target. Its flux measured with SEVIRI is then compared to a model that allows computing the total irradiance from the Moon disc for a given position of the observer and observation time, like ROLO (RObotic Lunar Observatory), GIRO (GSICS [Global Space-based Inter-Calibration System] Implementation of the ROLO model), or LIME (Lunar Irradiance Model of the European space agency). The scatter seen in ROLO lunar irradiance comparisons of multiple spacecraft instruments that have viewed the Moon amounts to 5-10\% [7], and one of the solar channels, NIR-016, cannot be analysed properly due to limitations of the GIRO/ROLO calibration reference [8]. LIME predicts $3 \%$ - 5\% higher disk integrated lunar irradiance than the GIRO/ROLO model for the visible and near-infrared channels [9]. In the light of these large uncertainties an alternative radiometric reference is desperately needed, in particular at $1.6 \mu \mathrm{m}$. Fortunately the Moon is not the only natural object outside the Earth's atmosphere that is within the dynamic range of most imaging instruments on Earth-orbiting spacecraft, as was claimed in the past [10]. Both Mercury and Venus give a good signal-to-noise ratio in most channels of SEVIRI as well, including the infrared. As all of the models of the Moon mentioned above cover only wavelengths below $2 \mu \mathrm{m}$, we also explored the potential of Mercury as calibration standard in the thermal infrared.

\section{Methodology}

$\mathbf{F}$ OR our investigation we had to identify those moments in time, when a planet was present in the images taken by SEVIRI. These are approximately squares with a length of $18.0^{\circ}$, where the Earth fills a circle with a diameter of $16.8^{\circ}$ and any other solar system object has to be close to the edge to be seen. Mercury has a high infrared radiance when close to superior conjunction, because in this case its subsolar point, and therefore its area of highest temperature, is near the center of its disk as seen by SEVIRI. It can provide SEVIRI with the largest flux of all planets at 4 and $6 \mu \mathrm{m}$, and Venus provides the largest flux at visible wavelengths - except for the Earth. Therefore we limited our search to appearances of
TABLE I

OBSERVATIONS OF PLANETS WITH SEVIRI ANALYSED BY US; THE FIRST COLUMN GIVES THE VERSION OF METEOSAT, THE SECOND COLUMN GIVES THE TARGET BODY, THE THIRD COLUMN GIVES THE DATE AND TIME (UT) OF THE END OF THE REPEAT CYCLE, THE FOURTH COLUMN GIVES THE APPARENT VISUAL MAGNITUDE OF THE TARGET BODY, THE FIFTH COLUMN GIVES THE EQUATORIAL ANGULAR WIDTH OF THE TARGET BODY FULL DISK, THE SIXTH COLUMN GIVES THE SOLAR ELONGATION ANGLE AND THE SEVENTH COLUMN GIVES THE PHASE ANGLE (SUN-PLANET-SEVIRI).

\begin{tabular}{|c|c|c|c|c|c|c|}
\hline MSG & Pl. & yyyy-m-dd, time & Mag & Diam & S-O-T & S-T-O \\
\hline 10 & $q$ & 2016-3-14 23:30 & -1.060 & $4.954 "$ & $8.44^{\circ}$ & $21.33^{\circ}$ \\
\hline 10 & $\emptyset$ & 2016-3-15 0:30 & -1.063 & 4.953” & $8.41^{\circ}$ & $21.26^{\circ}$ \\
\hline 10 & q & $2017-5-15$ 21:45 & -4.615 & $30.198 "$ & $44.31^{\circ}$ & $103.60^{\circ}$ \\
\hline 10 & $\emptyset$ & $2017-5-15$ 21:45 & 0.613 & $8.525 "$ & $25.66^{\circ}$ & $105.62^{\circ}$ \\
\hline 10 & 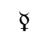 & $2017-5-15$ 22:00 & 0.613 & $8.523 "$ & $25.66^{\circ}$ & $105.60^{\circ}$ \\
\hline 10 & 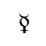 & $2017-5-15$ 22:15 & 0.612 & $8.521 "$ & $25.66^{\circ}$ & $105.57^{\circ}$ \\
\hline 10 & 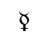 & $2017-5-15$ 22:30 & 0.612 & $8.520 "$ & $25.66^{\circ}$ & $105.55^{\circ}$ \\
\hline 10 & $\emptyset$ & $2017-5-15$ 22:45 & 0.611 & $8.518^{\prime \prime}$ & $25.66^{\circ}$ & $105.53^{\circ}$ \\
\hline 10 & q & $2017-5-17$ 21:45 & -4.592 & 29.367" & $44.66^{\circ}$ & $102.02^{\circ}$ \\
\hline 11 & $q$ & 2019-9-26 0:30 & -0.385 & $5.089 ”$ & $16.51^{\circ}$ & $38.36^{\circ}$ \\
\hline 11 & 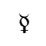 & 2019-9-26 1:30 & -0.383 & $5.090 "$ & $16.54^{\circ}$ & $38.42^{\circ}$ \\
\hline 11 & q & 2019-9-30 0:15 & -3.898 & $10.015^{\prime \prime}$ & $12.66^{\circ}$ & $17.68^{\circ}$ \\
\hline 11 & q & 2019-9-30 1:15 & -3.898 & 10.016" & $12.67^{\circ}$ & $17.70^{\circ}$ \\
\hline
\end{tabular}

these two planets. As Meteosat was in a geostationary orbit, its altitude was fixed at $35,786 \mathrm{~km}$, and its latitude at $0^{\circ}$. Only the longitude of the satellite is unconstrained, its exact value is available from EUMETSAT ${ }^{2}$. The results of our search are shown in Table I. This list is far from complete: There are many more observations of Mercury and Venus with SEVIRI on Meteosat-10 and -11 , not to mention other planets and weather satellites. The data sets used by us, however, are sufficient to demonstrate the value of planets for checking some aspects of the imaging and radiometric performance. 


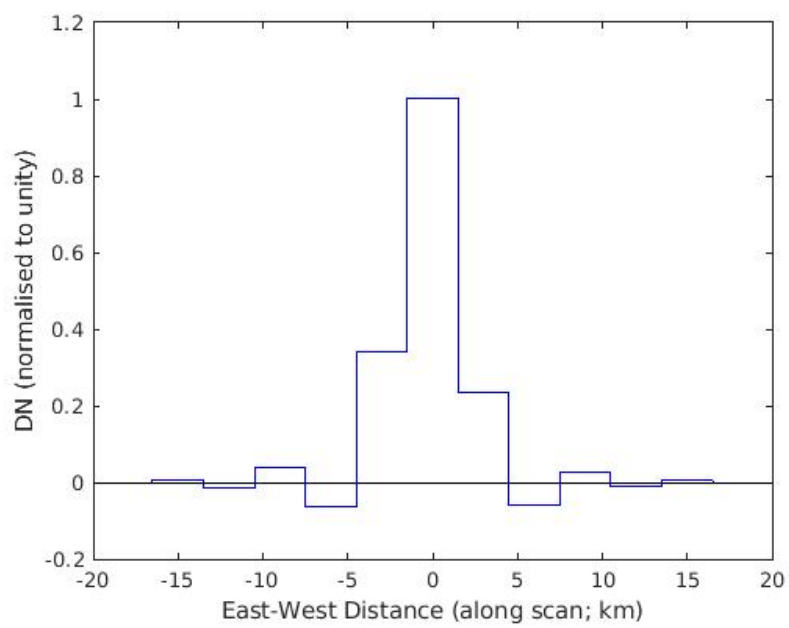

Fig. 2. The E-W signal distribution in observations of Mercury with IR-039 on $5 / 15,2017$. The planet was close to, but not exactly in, the center of a pixel. Note the negative side lobes of the finite impulse response applied to the Level 1.0 data.

Once a planet is found in an image from SEVIRI, we identified the pixel with the highest signal, i. e. counts. As Mercury is much smaller than the IFOV (Instantaneous Field Of View) of SEVIRI - we ignored the broad bandwidth channel - we could assume that it was fully included in this pixel, if it was close to the center of the diffraction pattern. Then we calculated the average counts from the pixels surrounding the planet in order to determine the signal from deep space, i. e. for zero incoming flux. This way we could establish the calibration offset for each band. Here one has to take into account the fact that the counts corresponding to deep space can vary within the full frame because of stray light. This phenomenon manifests itself in particular in the Sun eclipse season [2], and it is the reason why we only used pixels in the surroundings of the planet for establishing the zero level.

The central pixels of the image of the planet were then used to determine its exact position in the frame by fitting a Gaussian function in one variable - column or row - to the counts averaged over several pixels in the perpendicular direction, i. e. row or column, to improve the signal-to-noise ratio. In doing so we avoided all pixels with counts much lower than the signal from deep space. Such values are produced, when the finite impulse response (FIR) function is applied to Level 1.0 data. They are usually found two pixels away in EastWest direction from the center of the intensity distribution, see Figure 2. This figure agrees well with the convolution of the FIR filter and the SEVIRI point spread function used in the simulation of active fire observations on Earth in IR-039, see [11].

\section{RESULTS}

\section{A. Geometric Performances}

1) Relative accuracy within an image: Particularly interesting cases are those, where Mercury and Venus appear in the
TABLE II

Simultaneous observations of Mercury and Venus With SEVIRI ON 5/15, 2017 AT 21:45 (UT, END OF THE REPEAT CYCLE); THE FIRST COLUMN GIVES THE OBJECT, THE SECOND AND THIRD COLUMN GIVE THE COORDINATES OF THE PLANET, THE FOURTH COLUMN IDENTIFIES THE CHANNEL, THE FIFTH AND SIXTH COLUMN GIVE THE MEASURED POSITION OF THE PLANET ON THE IMAGE.

\begin{tabular}{|l|r|r|r|r|r|}
\hline Planet & R.A. & DEC & Channel & Column & Row \\
\hline Venus & $0: 44: 33.5$ & $3: 28: 34$ & VIS-006 & 120.8 & 2727.1 \\
Venus & $0: 40: 33.5$ & $3: 28: 34$ & NIR-008 & 102.7 & 2727.2 \\
Venus & $0: 40: 33.5$ & $3: 28: 34$ & NIR-016 & 138.8 & 2727.1 \\
Venus & $0: 40: 33.5$ & $3: 28: 34$ & WV-062 & 99.5 & 2745.0 \\
Venus & $0: 40: 33.5$ & $3: 28: 34$ & WV-073 & 81.6 & 2744.8 \\
Venus & $0: 40: 33.5$ & $3: 28: 34$ & IR-087 & 117.5 & 2745.0 \\
Venus & $0: 40: 33.5$ & $3: 28: 34$ & IR-108 & 135.4 & 2744.8 \\
Mercury & $1: 54: 35.7$ & $8: 12: 03$ & NIR-016 & 3831.5 & 3661.4 \\
Mercury & $1: 54: 35.7$ & $8: 12: 03$ & IR-039 & 3828.5 & 3679.1 \\
Mercury & $1: 54: 35.7$ & $8: 12: 03$ & WV-062 & 3792.5 & 3679.3 \\
Mercury & $1: 54: 35.7$ & $8: 12: 03$ & WV-073 & 3774.7 & 3679.2 \\
Mercury & $1: 54: 35.7$ & $8: 12: 03$ & IR-087 & 3810.5 & 3679.2 \\
Mercury & $1: 54: 35.7$ & $8: 12: 03$ & IR-108 & 3828.5 & 3679.0 \\
\hline
\end{tabular}

same image, because they allow to check for image distortion. Both planets could be identified for example in the image taken at $21: 45$ on $5 / 15,2017$, with SEVIRI on Meteosat-10. The exact positions of the planets in the images from different filters are given in Table II for the channels with a strong enough signal. The channels IR-039 and IR-108 have the same position in the focal plane, so Mercury should be at the same position in either image. For the SEVIRI focal planes layout see [12] and for a list of the different channels see Table III. Observations of the planets with the HRV (High Resolution Visible) channel were not available, and Mercury was too faint at wavelengths below one micron, therefore these images are not included. From the scatter of the measured distances between Mercury and Venus in the various channels one can conclude that the uncertainty of the positions obtained with our method is of the order 0.1 pixel, corresponding to $0.3 \mathrm{~km}$ on Earth at SSP (Sub-Satellite Point).

According to JPL's HORIZONS system ${ }^{3}$, the distance between Mercury and Venus from the vantage point of Meteosat10 was $18.308^{\circ}$. For calculating this number we took into consideration that Venus appeared in a scan that happened $3 \mathrm{~min}$ and $7 \mathrm{sec}$ earlier than the one containing Mercury, based on a spin rate of 100 rounds per minute and 934/3 satellite revolutions happening between these two scans. Obviously the satellite moved around the Earth during this time, and the viewing direction of SEVIRI changed accordingly. The distance between the two planets in the image amounts to 3809.3 pixels. Assuming an angular distance $d$ between two pixels of

$$
d=\arctan \left(\frac{s}{a}\right)=\left(4.8032 \cdot 10^{-3}\right)^{\circ}=17.292^{\prime \prime}
$$

where

$s=$ sampling $=3 \mathrm{~km}$

$a=$ altitude $=35,786 \mathrm{~km}$

$$
{ }^{3} \text { https://ssd.jpl.nasa.gov/horizons.cgi }
$$


TABLE III

DETAILED CHARACTERISTICS OF THE NARROW CHANNELS OF SEVIRI

[13]: CENTRAL WAVELENGTH, WAVELENGTH INTERVAL, AND ABBREVIATION OF THE BAND. THE LAST COLUMN TELLS WHETHER MERCURY OR VENUS CAN BE DETECTED WITH MORE THAN A HUNDRED COUNTS IN A GIVEN CHANNEL.

\begin{tabular}{|c|c|c|c|c|}
\hline Channel & Central $\lambda / \mu \mathrm{m}$ & $\lambda$ interval $/ \mu \mathrm{m}$ & Band & Planet \\
\hline 1 & 0.635 & $0.56-0.71$ & VIS-006 & q \\
\hline 2 & 0.81 & $0.74-0.88$ & VIS-008 & $q$ \\
\hline 3 & 1.64 & $1.5-1.78$ & NIR-016 & q \\
\hline 4 & 3.9 & $3.48-4.36$ & IR-039 & 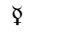 \\
\hline 5 & 6.25 & $5.35-7.15$ & WV-062 & $q, q$ \\
\hline 6 & 7.35 & $6.85-7.85$ & WV-073 & $\wp, q$ \\
\hline 7 & 8.7 & $8.3-9.1$ & IR-087 & $\Varangle, \uparrow$ \\
\hline 8 & 9.66 & $9.38-9.94$ & IR-097 & $\wp, \uparrow$ \\
\hline 9 & 10.8 & $9.8-11.8$ & IR-108 & $\Varangle, q$ \\
\hline 10 & 12 & $11-13$ & IR-120 & $q, q$ \\
\hline 11 & 13.4 & $12.4-14.4$ & IR-134 & $\wp, q$ \\
\hline
\end{tabular}

we determine the angular separation between the two planets as $18.297^{\circ}$ from the image, i. e. about $2.3 \times d$ less than we got from HORIZONS. The relevant requirement states that the relative accuracy within an image of 500 samples shall be of less than $3 \mathrm{~km}$ SSP (one pixel SSP), according to [2]. Given the much larger image size used by us, SEVIRI is compliant.

Agreement can be established between the angular distance between Venus and Mercury as measured by SEVIRI and the distance given by HORIZONS, if we assume a sampling distance of $3.0018 \mathrm{~km}$ instead of $3 \mathrm{~km}$.

2) Relative accuracy between consecutive images and tilt of the field of view: On May 15, 2017, Mercury was slightly more than $8^{\circ}$ north of the celestial equator. This means that it was observed five times in a row with SEVIRI, because it moved through the narrow gap between the North polar region of the Earth and the edge of the field of view of SEVIRI. Hence we could determine its exact position in five consecutive images, and the results are listed in Table IV. For our study we only used the four channels with the strongest signal in order to achieve maximum accuracy of the measurements; these channels are all in the thermal infrared.

Mercury's declination increased by 40 " between the first and the last image taken by SEVIRI, this is $2.3 \times d$. The difference in row number between the position of Mercury in the first and the last image, when averaged over all four channels, is $2.2 \pm 0.1$, i. e. we find agreement within the uncertainty of the measurement. Mercury's right ascension increased by $9.3^{s}=0.039^{\circ}$ between the first and the last image taken by SEVIRI. Taking the fact into account that the satellite had moved with an angular velocity of $15^{\circ}$ per hour in its orbit around the Earth, we get an apparent motion of $14.961^{\circ}$ of the planet between the first and the last image, this is $3114.8 \times d$. The difference in column number between the position of Mercury in the first and the last image is $3113.5 \pm 0.5$, when the average of all channels is calculated. This difference can be explained by the uncertainty of the measurements and the upper limit of the image distortion calculated in the previous subsection. If the image contortion is actually just a wrong sampling distance, then the consecutive images of Mercury suggest a sampling distance of $3.0013 \pm 0.0004 \mathrm{~km}$ instead of $3 \mathrm{~km}$.

In any case we can only make an estimate of the upper limit of the tilt $t$ of the rows in the images of SEVIRI against the equator:

$$
t \leq \arctan \left(\frac{0.2}{3114}\right)=\left(3.7 \cdot 10^{-3}\right)^{\circ}=13
$$

0.2 pixels is the maximum difference between the actual movement of Mercury in North-South direction (declination, 2.3 pixels per hour) and the value measured with SEVIRI ( $2.2 \pm 0.1$ pixels per hour) when allowing for its uncertainty.

If consecutive images had a perfect relative accuracy, Mercury's position would differ by the same amount in row (0.55) and column number (-778.4) from one image to the next. This is not the case with the actually measured positions of Mercury with Meteosat-10, and the deviations from the expected position in each image can be used to determine the relative accuracy. The results are listed in Table IV, from which we derive a standard deviation of 0.14 pixel or $0.42 \mathrm{~km}$. This value is only an upper limit of the relative accuracy of the position of a pixel in SEVIRI, because our method of measuring the position of Mercury in the image has also an uncertainty. We were mindful of Mercury's apparent position being very close to the Earth at 22:15, i. e. in the third image. The path of a light beam from Mercury to Meteosat led in this case through the stratosphere, where it was subjected to refraction and absorption. These effects caused probably the slight, northward shift of its position, i. e. towards higher row number, see Table IV, and less digital counts after background subtraction, see the last column of Table VII. These peculiarities, however, could also be due to stray light brought about by nearby Earth, therefore we excluded the problem case at 22:15 from the calculation of the standard deviation.

The relevant requirement states that the relative accuracy between two consecutive images shall be of less than $1.2 \mathrm{~km} \mathrm{SSP} \mathrm{(sub-satellite} \mathrm{point),} \mathrm{according} \mathrm{to} \mathrm{[2].} \mathrm{Although}$ we have only few consecutive images, we feel confident that SEVIRI is compliant. Its accuracy is probably two or even three times as good as required.

3) Mis-registration of the same pixel in different channels: With the values in Table IV we also searched for residuals in the relative inter-channel geometrical accuracy of the IR/WV focal plane. The different channels point at positions that are 18 pixels apart in the East-West direction, with WV-073/IR-134 being most western and IR-039/IR-108 being most eastern [12]. This arrangement is obvious in the numbers of the columns in Table IV for the different channels 
TABLE IV

CONSECUTIVE OBSERVATIONS OF MERCURY WITH SEVIRI ON 5/15, 2017; THE FIRST COLUMN GIVES THE TIME OF THE END OF THE REPEAT CYCLE, THE SECOND AND THIRD COLUMN GIVE THE COORDINATES OF THE PLANET AT THE END OF THE REPEAT CYCLE, THE FOURTH COLUMN IDENTIFIES THE CHANNEL, THE FIFTH AND SIXTH COLUMN GIVE THE MEASURED POSITION OF THE PLANET IN THE IMAGE.

\begin{tabular}{|r|r|r|r|r|r|}
\hline Time & R.A. & DEC & Channel & Column & Row \\
\hline $21: 45$ & $1: 54: 35.7$ & $8: 12: 03$ & IR-039 & 3828.5 & 3679.1 \\
$22: 00$ & $1: 54: 37.9$ & $8: 12: 13$ & IR-039 & 3050.1 & 3679.6 \\
$22: 15$ & $1: 54: 40.3$ & $8: 12: 23$ & IR-039 & 2272.0 & 3680.4 \\
$22: 30$ & $1: 54: 42.7$ & $8: 12: 33$ & IR-039 & 1493.2 & 3680.7 \\
$22: 45$ & $1: 54: 45.0$ & $8: 12: 43$ & IR-039 & 715.0 & 3681.4 \\
$21: 45$ & $1: 54: 35.7$ & $8: 12: 03$ & WV-062 & 3792.5 & 3679.3 \\
$22: 00$ & $1: 54: 37.9$ & $8: 12: 13$ & WV-062 & 3014.1 & 3679.7 \\
$22: 15$ & $1: 54: 40.3$ & $8: 12: 23$ & WV-062 & 2236.0 & 3680.5 \\
$22: 30$ & $1: 54: 42.7$ & $8: 12: 33$ & WV-062 & 1457.3 & 3680.7 \\
$22: 45$ & $1: 54: 45.0$ & $8: 12: 43$ & WV-062 & 679.0 & 3681.4 \\
$21: 45$ & $1: 54: 35.7$ & $8: 12: 03$ & WV-073 & 3774.7 & 3679.2 \\
$22: 00$ & $1: 54: 37.9$ & $8: 12: 13$ & WV-073 & 2996.0 & 3679.6 \\
$22: 15$ & $1: 54: 40.3$ & $8: 12: 23$ & WV-073 & 2217.7 & 3680.3 \\
$22: 30$ & $1: 54: 42.7$ & $8: 12: 33$ & WV-073 & 1439.5 & 3680.7 \\
$22: 45$ & $1: 54: 45.0$ & $8: 12: 43$ & WV-073 & 660.9 & 3681.3 \\
$21: 45$ & $1: 54: 35.7$ & $8: 12: 03$ & IR-087 & 3810.5 & 3679.2 \\
$22: 00$ & $1: 54: 37.9$ & $8: 12: 13$ & IR-087 & 3032.2 & 3679.7 \\
$22: 15$ & $1: 54: 40.3$ & $8: 12: 23$ & IR-087 & 2253.8 & 3680.3 \\
$22: 30$ & $1: 54: 42.7$ & $8: 12: 33$ & IR-087 & 1475.6 & 3680.8 \\
$22: 45$ & $1: 54: 45.0$ & $8: 12: 43$ & IR-087 & 697.1 & 3681.4 \\
\hline
\end{tabular}

from the same repeat cycle.

Mercury is too faint for the determination of its exact position in the focal plane at wavelengths below $2 \mu \mathrm{m}$. Therefore we had to use observations of Venus for the VIS/NIR range, see Table $\mathrm{V}$. The numbers of the columns in this table prove that VIS-008 is most western and NIR-016 is most eastern. (We did not include the High Resolution Visible Channel in our study.) They confirm the SEVIRI nominal focal plane layout according to [12], whereas [4] give wrong positions for VIS-006 and VIS-008.

The errors of the co-registration of the channels in either SEVIRI focal plane are given in Table VI. As we have rather few observations of each planet, we can only give rough estimates of the pointing error. Digitization noise and the fact that not more than $3 \times 3$ or $4 \times 4$ pixels received enough flux from a planet to be useful for a calculation of its exact position, contributed to the overall uncertainty as well. The relevant requirement states that the maximum residual mis-registration shall be of less than 0.6 (0.75) $\mathrm{km}$ SSP (sub-satellite point) for the visible and near infra-red (water vapor and infrared) group of channels according to [2]. In spite of the uncertainties we may safely conclude that this requirement is fulfilled for SEVIRI on both Meteosat-10 and -11 . When considering the co-registration of channels on different focal planes, however, we find larger errors. As Venus provides enough flux in both warm and cold channels to determine its position, we can determine the relative positions of images from different focal
TABLE V

OBSERVATIONS OF VENUS WITH SEVIRI; THE FIRST COLUMN GIVES THE DATE AND TIME (UT) OF THE END OF THE REPEAT CYCLE, THE SECOND AND THIRD COLUMN GIVE THE COORDINATES OF VENUS AT THE END OF THE REPEAT CYCLE, THE FOURTH COLUMN IDENTIFIES THE CHANNEL, THE FIFTH AND SIXTH COLUMN GIVE THE MEASURED POSITION OF THE PLANET IN THE IMAGE.

\begin{tabular}{|r|r|r|r|r|r|}
\hline Date and Time & R.A. & DEC & Ch. & Col. & Row \\
\hline 5/15, 2017, 21:45 & $0: 40: 33.5$ & $3: 28: 34$ & 006 & 120.7 & 2727.1 \\
5/15, 2017, 21:45 & $0: 40: 33.5$ & $3: 28: 34$ & 008 & 102.7 & 2727.2 \\
5/15, 2017, 21:45 & $0: 40: 33.5$ & $3: 28: 34$ & 016 & 138.8 & 2727.1 \\
5/15, 2017, 21:45 & $0: 40: 33.5$ & $3: 28: 34$ & 062 & 99.5 & 2745.0 \\
5/15, 2017, 21:45 & $0: 40: 33.5$ & $3: 28: 34$ & 073 & 81.6 & 2744.8 \\
5/15, 2017, 21:45 & $0: 40: 33.5$ & $3: 28: 34$ & 087 & 117.5 & 2745.0 \\
5/15, 2017, 21:45 & $0: 40: 33.5$ & $3: 28: 34$ & 097 & 99.7 & 2745.0 \\
5/15, 2017, 21:45 & $0: 40: 33.5$ & $3: 28: 34$ & 108 & 135.4 & 2744.8 \\
5/15, 2017, 21:45 & $0: 40: 33.5$ & $3: 28: 34$ & 120 & 117.4 & 2744.9 \\
5/15, 2017, 21:45 & $0: 40: 33.5$ & $3: 28: 34$ & 134 & 81.6 & 2744.9 \\
5/17, 2017, 21:45 & $0: 46: 44.4$ & $3: 53: 56$ & 006 & 6.7 & 2815.6 \\
5/17, 2017, 21:45 & $0: 46: 44.4$ & $3: 53: 56$ & 016 & 24.8 & 2815.6 \\
5/17, 2017, 21:45 & $0: 46: 44.4$ & $3: 53: 56$ & 039 & 21.5 & 2833.8 \\
5/17, 2017, 21:45 & $0: 46: 44.4$ & $3: 53: 56$ & 108 & 21.2 & 2833.5 \\
9/30, 2019, 0:15 & $13: 11: 15.8$ & $-6: 31: 49$ & 006 & 3587.8 & 616.3 \\
9/30, 2019, 0:15 & $13: 11: 15.8$ & $-6: 31: 49$ & 008 & 3569.7 & 616.3 \\
9/30, 2019, 0:15 & $13: 11: 15.8$ & $-6: 31: 49$ & 016 & 3605.9 & 616.4 \\
9/30, 2019, 0:15 & $13: 11: 15.8$ & $-6: 31: 49$ & 062 & 3550.6 & 634.7 \\
9/30, 2019, 0:15 & $13: 11: 15.8$ & $-6: 31: 49$ & 073 & 3532.5 & 634.5 \\
9/30, 2019, 0:15 & $13: 11: 15.8$ & $-6: 31: 49$ & 087 & 3568.8 & 634.6 \\
9/30, 2019, 0:15 & $13: 11: 15.8$ & $-6: 31: 49$ & 097 & 3550.8 & 634.9 \\
9/30, 2019, 0:15 & $13: 11: 15.8$ & $-6: 31: 49$ & 108 & 3586.6 & 634.6 \\
9/30, 2019, 0:15 & $13: 11: 15.8$ & $-6: 31: 49$ & 120 & 3568.6 & 634.6 \\
9/30, 2019, 0:15 & $13: 11: 15.8$ & $-6: 31: 49$ & 134 & 3532.6 & 634.5 \\
9/30, 2019, 1:15 & $13: 11: 27.9$ & $-6: 33: 04$ & 006 & 485.6 & 629.8 \\
9/30, 2019, 1:15 & $13: 11: 27.9$ & $-6: 33: 04$ & 008 & 467.5 & 629.8 \\
9/30, 2019, 1:15 & $13: 11: 27.9$ & $-6: 33: 04$ & 016 & 503.7 & 629.9 \\
9/30, 2019, 1:15 & $13: 11: 27.9$ & $-6: 33: 04$ & 062 & 464.4 & 648.4 \\
9/30, 2019, 1:15 & $13: 11: 27.9$ & $-6: 33: 04$ & 073 & 446.3 & 648.3 \\
9/30, 2019, 1:15 & $13: 11: 27.9$ & $-6: 33: 04$ & 087 & 482.3 & 648.2 \\
9/30, 2019, 1:15 & $13: 11: 27.9$ & $-6: 33: 04$ & 097 & 464.3 & 648.5 \\
9/30, 2019, 1:15 & $13: 11: 27.9$ & $-6: 33: 04$ & 108 & 500.4 & 648.3 \\
9/30, 2019, 1:15 & $13: 11: 27.9$ & $-6: 33: 04$ & 120 & 482.5 & 648.3 \\
9/30, 2019, 1:15 & $13: 11: 27.9$ & $-6: 33: 04$ & 134 & 446.3 & 648.2 \\
\hline
\end{tabular}

planes with the numbers in Table V. The results are listed in the last rows of Table VI.

4) Jitter among scan lines: [2] reported the existence of an East-West "jitter". This phenomenon manifested itself massively in those of our observations, which happened within one hour of midnight. The "jumps" of a scan line can be so large that the channels at the extreme positions in the focal plane, for example WV-073 and IR-134, do not cover the whole Earth any more. This phenomenon can cause quite wrong flux values for dozens of pixels in the Level 1.5 data, simply because of missing data. Smaller manifestations of the jitter, however, are corrected for in the routine processing of the raw data. 
TABLE VI

IMAGE PERFORMANCE; THE FIRST THROUGH THIRD ROW GIVE THE IMAGE TO IMAGE ERROR. ALL MEASURED PERFORMANCES ARE WELL WITHIN SPECIFICATION (IN BOLD). THE FOURTH THROUGH NINTH ROW GIVE THE INTER-CHANNEL SPATIAL REgistration ERROR. THE MEASUREMENTS HERE SOMETIMES EXCEED THE SPECIFICATION. HOWEVER, THIS IS PROBABLY DUE TO EAST-WEST IMAGE JUMPS IN THE RAW DATA. THE SYSTEMATIC DIFFERENCES BETWEEN THE FOCAL PLANES IN THE NORTH-SOUTH DIRECTION ARE WITHIN SPECIFICATION TOO. ALL ERRORS ARE DEVIATIONS FROM THE NOMINAL POSITIONS AS GIVEN IN [12] AND ARE VALID FOR THE SUB-SATELLITE POINT.

\begin{tabular}{|l|l|l|l|l|}
\hline & \multicolumn{2}{|c|}{ MET-10 } & \multicolumn{2}{c|}{ MET-11 } \\
\hline \hline Group & $\begin{array}{l}\text { E-W } \\
\text { (km) }\end{array}$ & $\begin{array}{l}\text { N-S } \\
\text { (km) }\end{array}$ & $\begin{array}{l}\text { E-W } \\
\text { (km) }\end{array}$ & $\begin{array}{l}\text { N-S } \\
\text { (km) }\end{array}$ \\
\hline Im.-im. error (spec) & $\mathbf{1 . 2}$ & $\mathbf{1 . 2}$ & $\mathbf{1 . 2}$ & $\mathbf{1 . 2}$ \\
Im.-im. error (mean) & 0.24 & 0.43 & & \\
Im.-im. error (max) & 0.39 & 0.68 & & \\
\hline VIS/NIR co-reg. (spec) & $\mathbf{0 . 6}$ & $\mathbf{0 . 6}$ & $\mathbf{0 . 6}$ & $\mathbf{0 . 6}$ \\
VIS/NIR co-reg. (mean) & 0.17 & 0.17 & 0.3 & 0.17 \\
VIS/NIR co-reg. (max) & 0.3 & 0.3 & 0.6 & 0.3 \\
039-087 co-reg. (spec) & $\mathbf{0 . 7 5}$ & $\mathbf{0 . 7 5}$ & $\mathbf{0 . 7 5}$ & $\mathbf{0 . 7 5}$ \\
039-087 co-reg. (mean) & 0.4 & 0.2 & 0.3 & 0.4 \\
039-087 co-reg. (max) & 1.2 & 0.6 & 0.9 & 0.9 \\
\hline Focal planes co-reg. (spec) & $\mathbf{n . ~ a . ~}$ & $\mathbf{1 . 5}$ & n. a. & $\mathbf{1 . 5}$ \\
Focal planes co-reg. (mean) & 9.61 & -0.66 & 9.73 & 1.44 \\
Uncertainty & \pm 0.16 & \pm 0.14 & \pm 0.19 & \pm 0.16 \\
\hline
\end{tabular}

\section{B. Radiometric Performances}

The multiple observations of Mercury on 5/15, 2017, provide an opportunity to derive an upper limit for the short term radiometric error performance, because the flux from this planet does not change significantly within one hour, see the column "Mag" in Table I. In Table VII we have listed two values indicative of the flux of Mercury: The number of counts in the pixel getting the highest flux and the sum of the counts in all pixels getting flux from this planet. In the latter case we have subtracted the number of counts corresponding to no flux at all, i. e. pointing at deep space, away from any source.

1) Deep-Space Scans: The number of counts from the space corners is the offset Cal $_{\text {Offset }}$ and its nominal value can be calculated from the calibration coefficients in the level 1.5 file:

$$
C a l_{O f f s e t}=-\frac{\text { rad.BB_Processing_Offset }}{\text { rad.BB_Processing_Slope }}=51
$$

These calibration coefficients were the same for the infrared channels $(\lambda \geq 3 \mu \mathrm{m})$ in all files from Metop-10 we looked at, and $C a l_{O f f s e t}$ was always calculated as 51. Nevertheless the average $C_{\text {Caffset }}$ in the pixels surrounding Mercury differs sometimes by up to half a percent from this nominal value, probably due to low frequency noise problems [8]. The offset measured with pixels near Mercury was even larger than 52 at $22: 15$ on $5 / 15,2017$. This is because at that time Mercury came closest to Earth as seen from the position of the spacecraft, and the counts were increased by stray light from the Earth or Sun light scattered in the highest layer of the Earth's atmosphere. With Meteosat-11 we found a
TABLE VII

CONSECUTIVE OBSERVATIONS OF MERCURY WITH SEVIRI ON 5/15, 2017; THE FIRST COLUMN GIVES THE TIME OF THE END OF THE REPEAT CYCLE, THE SECOND COLUMN IDENTIFIES THE CHANNEL, THE THIRD COLUMN GIVES THE COUNTS OF THE BRIGHTEST PIXEL IN THE IMAGE OF MERCURY (WITHOUT BACKGROUND SUBTRACTION), THE FOURTH COLUMN GIVES THE AVERAGE COUNTS FROM DEEP SPACE VIEWINGS AWAY FROM MERCURY, THE FIFTH COLUMN GIVES THE SUM OF ALL PIXELS RECEIVING FLUX FROM MERCURY AFTER BACKGROUND SUBTRACTION.

\begin{tabular}{|r|r|r|r|r|}
\hline Time & IR Channel & Maximum & Background & Total \\
\hline UT & & counts & counts & counts \\
\hline 21:45 & 039 & 364 & $51.06 \pm 0.09$ & 832 \\
$22: 00$ & 039 & 385 & $51.17 \pm 0.06$ & 822 \\
$22: 15$ & 039 & 396 & $52.47 \pm 0.02$ & 750 \\
$22: 30$ & 039 & 360 & $51.24 \pm 0.06$ & 783 \\
$22: 45$ & 039 & 424 & $51.26 \pm 0.06$ & 824 \\
$21: 45$ & 062 & 245 & $50.84 \pm 0.03$ & 533 \\
$22: 00$ & 062 & 274 & $50.91 \pm 0.04$ & 530 \\
$22: 15$ & 062 & 262 & $53.39 \pm 0.04$ & 474 \\
$22: 30$ & 062 & 256 & $51.00 \pm 0.04$ & 532 \\
$22: 45$ & 062 & 267 & $51.02 \pm 0.05$ & 526 \\
$21: 45$ & 073 & 198 & $51.18 \pm 0.07$ & 367 \\
$22: 00$ & 073 & 206 & $51.09 \pm 0.07$ & 392 \\
$22: 15$ & 073 & 205 & $52.91 \pm 0.03$ & 330 \\
$22: 30$ & 073 & 197 & $51.13 \pm 0.04$ & 372 \\
$22: 45$ & 073 & 206 & $51.20 \pm 0.06$ & 376 \\
$21: 45$ & 087 & 105 & $51.20 \pm 0.05$ & 166 \\
$22: 00$ & 087 & 120 & $51.24 \pm 0.04$ & 170 \\
$22: 15$ & 087 & 115 & $52.88 \pm 0.05$ & 126 \\
$22: 30$ & 087 & 114 & $50.99 \pm 0.05$ & 174 \\
$22: 45$ & 087 & 113 & $51.14 \pm 0.04$ & 164 \\
\hline
\end{tabular}

background of 102 counts for channels WV-062 and WV-073.

2) Radiance of Mercury: The calibration coefficients as given in the level 1.5 files (rad.BB_Processing_Offset and rad.BB_Processing_Slope) were then used to convert counts to radiance and further, with the Planck function, to brightness temperature, using the apparent diameter of Mercury. As starting point we took the highest counts in a single pixel, assuming that Mercury was fully included in the field of view of SEVIRI, because it is a target body small in size, see the column "Diam" in Table I. This field of view is rhombus-shaped, and its sides have a length of $4.8 \mathrm{~km}$ or 27.7" according to [12]. [6] claims a length of $5 \mathrm{~km}$, and the CMSAF web page gives $4 \mathrm{~km}^{4}$. This means that Mercury is always smaller than the field of view, but Venus can be larger.

3) A Model of Mercury's Radiant Flux: In Figure 3 we compare the flux values from the observations of Mercury on Meteosat-11 from 9/26, 2019, with the predictions from a thermophysical model [14] using Mercury's known physical and thermal properties [15], [16], [17]. Channel IR-039 was saturated, therefore it is not included in the plot, but the counts in the non-saturated pixels provide consistent flux densities for most channels. Only a small number of counts is

\footnotetext{
${ }^{4}$ https://wui.cmsaf.eu/safira/action/viewDoiDetails? acronym=CMA_SEVIRI_V001
} 


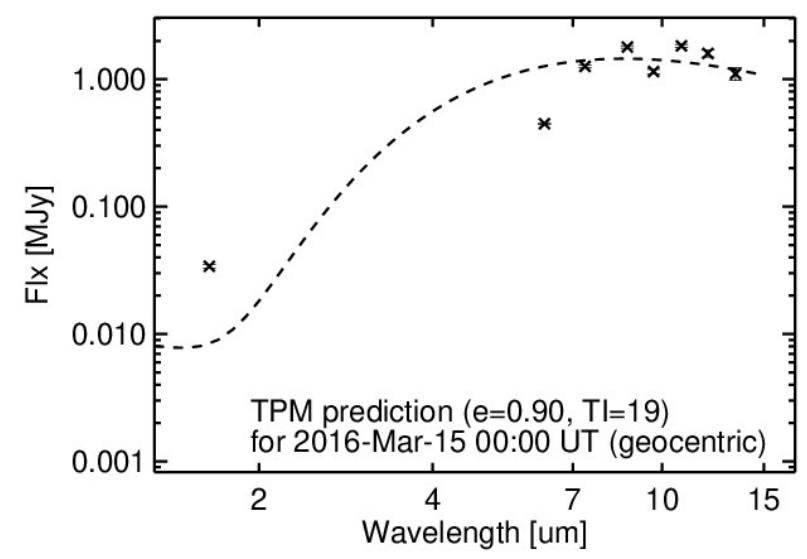

Fig. 3. Flux density of Mercury as measured with SEVIRI on 3/15, 2016 (crosses with error bars) and prediction of a thermophysical model, using a lunar-like surface roughness and a thermal inertia (TI) of $19 \mathrm{~J} \mathrm{~m}^{-2} \mathrm{~K}^{-1} \mathrm{~s}^{-0.5}$ [15].

produced in channel NIR-016, and it is difficult to model the mixture of reflected sunlight and Mercury's thermal emission at this wavelength. Channel WV-062 is particularly interesting, because absorption by atmospheric water vapour makes this wavelength inaccessible to ground-based telescopes. The measurement suggests that Mercury's emissivity is lower at $6.2 \mu \mathrm{m}$ than the emissivity of 0.9 assumed in the model. This finding is supported by a steep decline of emissivity from 5 to $6 \mu \mathrm{m}$, which is present in a spectrum obtained with the NASA Infrared Telescope Facility on Mauna Kea, Hawaii [18]. Another emissivity dip may be present at $9.7 \mu \mathrm{m}$, the possible wavelength of a Christiansen feature [19].

\section{Discussion}

$\mathbf{S}$ EVERAL examples were given to demonstrate the usefulness of bright point sources for the calibration of SEVIRI. Unlike the Moon and landmarks on Earth, they provide strong flux at a well-defined position surrounded by deep space. Therefore they are particularly useful for checking requirements on the geometric performance of the instrument in flight. In the following we give a summary of what we learned about SEVIRI on Metop-10 and -11 .

\section{A. Geometric quality}

- The relative accuracy between two consecutive images was investigated with a series of five consecutive images of Mercury, taking the movements of the weather satellite and the planet into account. The values in our Table VI suggest a slightly larger error in the N-S than in the E-W direction, but this might simply be a consequence of the signal being smeared across many pixels along the $\mathrm{x}$-axis [11], whereas in the $\mathrm{y}$-axis only two or three pixels were available to deduce the position of the planet from. As the planet changed its position considerably in consecutive images, any tilt of the scan direction against the celestial equator, if it existed, would contribute to the error in the N-S direction as well. In the light of these uncertainties our estimates in Table VI are compatible with the MSG-1 satellite commissioning [20], viz. mean error $\approx 0.33 \mathrm{~km}$ and $\max$ error $\approx 0.66 \mathrm{~km}$ in both $\mathrm{N}-\mathrm{S}$ and $\mathrm{E}-\mathrm{W}$.

- The relative accuracy within an image was investigated with a simultaneous appearance of Mercury and Venus in an image. We note that the apparent distance between Mercury and Venus in our example is larger than the diameter of the Earth as seen by SEVIRI, hence we checked the image distortion on a scale that cannot be achieved with landmarks on Earth. If we assume that the relative accuracy within an image is perfect, then the discrepancy between the distance of Mercury and Venus in the image from SEVIRI and the distance given by HORIZONS implies that the sampling for narrow channels is $3.0018 \mathrm{~km}$ instead of $3 \mathrm{~km}$ at SSP. A somewhat lower value was obtained from analysing the movement of Mercury in the five consecutive observations of this planet on 5/15, 2017, see Section III-A2. We conclude therefore that the sampling is $3.0016 \pm 0.0003 \mathrm{~km}$.

- For checking the maximum residual mis-registration we have compared the exact position of Venus in the visible and near infra-red group of channels of both Metop-10 and -11 , because this is the brightest planet in reflected sunlight, apart from Earth. The results are given in our Table VI. The mis-registration was never larger than $0.6 \mathrm{~km}$ at SSP, after taking the different positions of the detectors in the focal plane into account (Table I). As Venus is not a point source, our measurements provide only an upper limit of the actual mis-registration.

There is an analogous requirement for the remaining channels, and compliance was checked with Mercury. This requirement is stated without physical unit in [2], so we assume a maximum allowed mis-registration of $0.75 \mathrm{~km}$ at SSP according to [1]. The measured mis-registration was in fact larger than $0.75 \mathrm{~km}$ at SSP in certain cases, but this was probably a consequence of East-West image jumps that always affect three consecutive detector lines in the same way and that happen often during eclipses [2], i. e. close in time to some of our observations. They can make an existing mis-registration worse, when they cause shifts between different parts of the image. Hence our measurements do not necessarily prove an occasional non-compliance withthe requirement.

- The residual mis-registration of the same pixel in different focal planes can be much larger than the mis-registration within either focal plane described in the previous bullet point.

Recently, a structured residual error was found for certain channel pairs of SEVIRI on Meteosat-11, when the inter-channel relative accuracy was measured using 
direct correlation of image channels [4]. As this was the first time that the pointing table of SEVIRI had to be corrected during the operational phase, it is interesting to search for mis-registration between the VIS/NIR and the IR/WV focal plane with Venus. Choosing a planet for this check avoids a major drawback of the matching method employed by [4], namely the fact that the same feature might look different in different channels. A planet is a small disk at the same position in the sky for every channel with a much stronger contrast to its neighbourhood than landmarks.

It follows from the numbers in Table VI that there is a shift of almost $10 \mathrm{~km}$ in E-W between the VIS/NIR and the IR/WV channels for both Meteosat-10 and -11 . This is no cause for concern, because even larger shifts between different detector lines happen as a consequence of East-West image jumps. They are corrected by processing beyond level 1.0. The anomaly described by [4], however, is caused by the too large distance between two groups of channels in the N-S direction for Meteosat-11. They found an inter-channel residual registration error of $0.9 \mathrm{~km}$ between VIS/NIR channels on the one hand and IR/WV channels on the other hand. Our measurements show that the distance between the two focal planes is $1.44 \pm 0.16 \mathrm{~km}$ larger for Meteosat-11 and $0.66 \pm 0.14 \mathrm{~km}$ smaller for Meteosat-10 than the nominal value of $18 \mathrm{~km}$. Hence our results support the conclusion of [4] that the inter channel registration errors for different focal planes are significant on METEOSAT-11. A similar conclusion was reached by [5]. In any case they amount to less than $3 \mathrm{~km} \mathrm{SSP}$ for all channels, i. e. they still comply with the requirement.

\section{B. Radiometric Accuracy}

If the planets have a distance of less than $9^{\circ}$ from the celestial equator, they can usually be found in two images with SEVIRI that were taken one hour apart: once to the east and once to the west of the Earth, see Table I. Assuming that the flux from the planets does not change within one hour, we interpret the difference between the counts from the narrow-bandwidth, warm channels in these two images as an upper limit of the radiometric stability of the instrument. Our values are compared in Table VIII to the accuracy of the vicarious calibration and the performance of four cold channels in the SEVIRI FM tests. The similarity of the results obtained with the traditional methods and ours suggests that extracting planetary irradiance is as accurate as measuring irradiance from pseudo invariant calibration sites, and that planets are suitable for the validation, calibration, and characterisation of visible infrared imagers. Mercury has a truly invariant surface, free of clouds or dust storms, and Venus is always in a shroud of clouds. Unfortunately the average albedo of Venus still needs to be established more accurately - it changes over annual and longer timescales [21], [22].
TABLE VIII

COMPARISON BETWEEN SHORT-TERM RADIOMETRIC ERROR REQUIREMENTS (FIFTH COLUMN) AND PERFORMANCES DERIVED FROM OBSERVATIONS OF MERCURY AND VENUS WITH SEVIRI (THIRD COLUMN). THE FOURTH COLUMN GIVES THE CALIBRATION UNCERTAINTY DERIVED FROM SEVIRI COUNT VALUES OVER THE DESERT [6] FOR VIS AND NIR AND FROM THE SEVIRI FM TEST RESULTS [12] FOR WV AND IR. THE LAST COLUMN GIVES THE RETRIEVED OFFSET VALUES. THEY ARE VERY CLOSE TO THE NOMINAL VALUE OF 51.

\begin{tabular}{|l|c|c|c|c|c|}
\hline Channel & Planet & perf & exp & req & Retr. Off. \\
\hline VIS-006 & $q$ & $4.8 \%$ & $4 \%$ & $0.5 \%$ & $51.00 \pm 0.01$ \\
\hline VIS-008 & $\wp$ & $2.0 \%$ & $4 \%$ & $0.5 \%$ & $51.00 \pm 0.01$ \\
\hline NIR-016 & $\wp$ & $5.3 \%$ & $5 \%$ & $1.0 \%$ & $51.02 \pm 0.03$ \\
\hline IR-039 & $\wp$ & $0.9 \%$ & $1.0 \%$ & $1.4 \%$ & $51.16 \pm 0.05$ \\
\hline WV-062 & $\wp$ & $1.3 \%$ & $1.5 \%$ & $2.8 \%$ & $50.93 \pm 0.03$ \\
\hline WV-073 & $\wp$ & $2.3 \%$ & $1.5 \%$ & $2.4 \%$ & $51.19 \pm 0.05$ \\
\hline IR-087 & $\wp$ & $1.3 \%$ & $0.3 \%$ & $0.5 \%$ & $51.17 \pm 0.03$ \\
\hline
\end{tabular}

\section{CONCLUSION}

As Mercury's diameter is always smaller than the field of view of SEVIRI, and Venus' diameter most of the time, these objects are very well suited for checking the geometric performance, in particular the band to band registration accuracy, which was recently found to be faulty with SEVIRI on Meteosat-11. Mercury and Venus allow the investigation of such anomalies with a phenomenal precision in the absence of special spacecraft manoeuvres and even after the end of the mission. The lastest Advanced Imagers, however, have much smaller IFOVs that SEVIRI, and that means that they are able to spatially resolve Venus. A star is therefore better suited for the calibration of their VIS/NIR channels. It must be close to the celestial equator and bright, with a well-known spectrum - we suggest Procyon, a Gaia benchmark star [23].

\section{ACKNOWLEDGMENT}

The authors would like to thank Pierre Drossart for information about the variability of Venus.

This research was funded by Deutsche Forschungsgemeinschaft, project number 421761264 (MW-Luna). With this work we contribute to the Cluster of Excellence "CLICCS-Climate, Climatic Change, and Society" funded by the Deutsche Forschungsgemeinschaft DFG (EXC 2037, Project Number 390683824), and to the Center for Earth System Research and Sustainability (CEN) of Universität Hamburg.

\section{REFERENCES}

[1] D. Just, SEVIRI LEVEL 1.5 DATA, Proceedings of the First MSG RAO Workshop, Bologna, Italy, May 17-19, 2000.

[2] C. Hanson, J. Mueller, Status of the SEVIRI Level 1.5 Data, Proceedings Second MSG RAO Workshop, Salzburg, Austria, Sept. 9-10, 2004.

[3] P. Pili, L. Matheson, C. Tranquilli, J. Müller, T. Hewison, S. Carlier, S. Bianchi, P. Coste, The In-orbit Performance of the Meteosat Second Generation SEVIRI Instruments, Proceedings of the EUMETSAT 2016 Meteorological Satellite Conference, Darmstadt, Germany, Sept. 26-30, 2016 
[4] J. Nain, J. Mueller, Improving band to band registration accuracy of SEVIRI level 1.5 products, Proc. SPIE 11155, Image and Signal Processing for Remote Sensing XXV, 1115503 (7 October 2019).

[5] V. Debaecker, S. Kocaman, S. Saunier, K. Garcia, S. Bas, D. Just, On the geometric accuracy and stability of MSG SEVIRI images, Atmospheric Environment, Volume 262, 118645, 2021.

[6] Y. M. Govaerts, M. Clerici, Operation Vicarious calibration of the MSG/SEVIRI Solar Channels, Proceedings of the EUMETSAT 2003 Meteorological Satellite Conference, Darmstadt, Germany, Sept. 29 - Oct. 3, 2003 .

[7] T. Stone, H. Kieffer, Assessment of uncertainty in ROLO lunar irradiance for on-orbit calibration, Proc. SPIE 5542, Earth Observing Systems IX, Pages 300-310, 2004.

[8] C. Tranquilli, B. Viticchie, S. Pessina, T. Hewison, J. Mueller, S. Wagner, Meteosat SEVIRI Performance Characterisation and Calibration with Dedicated Moon/Sun/Deep-space Scans, SpaceOps 2016 Conference, Daejeon, Korea, May 16-20, 2016.

[9] S. Taylor, S. Adriaensen, C. Toledano, A. Barreto, E. Woolliams, M. Bouvet, LIME, 23rd EGU General Assembly, held online 19-30 April, 2021.

[10] H. Kieffer, J. M. Anderson, Use of the moon for spacecraft calibration over 350 to $2500 \mathrm{~nm}$, Proc. SPIE 3498, Sensors, Systems, and NextGeneration Satellites II, Pages 325-336, 1998.

[11] M. J. Wooster, G. Roberts, P. H. Freeborn, W. Xu, Y. Govaerts, R. Beeby, J. He, A. Lattanzio, R. Mullen, Meteosat SEVIRI Fire Radiative Power products from the Land Surface Analysis Satellite Applications Facility, International Journal of Remote Sensing, Volume 30, Issue 17, Pages 4567-4579, 2009.

[12] P. Pili, SEVIRI INSTRUMENT LEVEL 1.0 DATA, Proceedings of the First MSG RAO Workshop, Bologna, Italy, May 17-19, 2000.

[13] M. J. Wooster, G. Roberts, P. H. Freeborn, W. Xu, Y. Govaerts, R. Beeby, J. He, A. Lattanzio, D. Fisher, R. Mullen, LSA SAF Meteosat FRP products, Atmospheric Chemistry and Physics, Volume 15, Pages 1321713239,2015

[14] T. G. Müller, M. Burgdorf, V. Alí-Lagoa, S. A. Buehler, M. Prange The Moon at thermal infrared wavelengths, Astronomy \& Astrophysics, Volume 650, A38, 2021.

[15] P. O. Hayne, J. L. Bandfield, M. A. Siegler, A. R. Vasavada, R. R. Ghent, J.-P. Williams, B. T. Greenhagen, O. Aharonson, C. M. Elder, P. G. Lucey, D. A. Paige Global Regolith Thermophysical Properties of the Moon From the Diviner Lunar Radiometer Experiment, Journal of Geophysical Research (Planets), Volume 122, Issue 12, Pages 2371-2400, 2017.

[16] B. Rozitis, S. F. Green, Directional characteristics of thermal-infrared beaming from atmosphereless planetary surfaces, Monthly Notices of the Royal Astronomical Society, Volume 415, Issue 3, Pages 2041-2062, 2011.

[17] J. L. Bandfield, P. O. Hayne, J.-P. Williams, B. T. Greenhagen, D. A. Paige Lunar surface roughness derived from LRO Diviner Radiometer observations, Icarus, Volume 248, Pages 357-372, 2015.

[18] A. L. Sprague, J. P. Emery, K. L. Donaldson, R. W. Russell, D. K. Lynch, A. L. Mazuk, Mercury, Meteoritics and Planetary Science, Volume 37, Pages 1255-1268, 2002.

[19] H. Hiesinger, J. Helbert, MERTIS Co-I Team, The Mercury Radiometer and Thermal Infrared Spectrometer for the BepiColombo mission, Planetary and Space Science, Volume 58, Number 102, Pages 144-165, 2010.

[20] D. M. A. Aminou, A. Ottenbacher, C. G. Hanson, P. Pili, J. Muller B. Blancke, B. Jacquet, S. Bianchi, P. Coste, F. Pasternak, F. Faure, Meteosat Second Generation, Proc. SPIE 5151, Earth Observing Systems VIII, Pages 599-608, 2003

[21] S. S. Limaye, D. Grassi, A. Mahieux, A. Migliorini, S. Tellmann, D. Titov, Venus Atmospheric Thermal Structure and Radiative Balance, Space Science Reviews, Volume 214, Number 102, 2018.

[22] Y. J. Lee, K.-L. Jessup, S. Perez-Hoyos, D. V. Titov, S. Lebonnois, J. Peralta, T. Horinouchi, T. Imamura, S. Limaye, E. Marcq, M. Takagi, A. Yamazaki, M. Yamada, S. Watanabe, S.-Y. Murakami, K. Ogohara, W. M. McClintock, G. Holsclaw, A. Roman, Long-term Variations of Venus's $365 \mathrm{~nm}$ Albedo Observed by Venus Express, Akatsuki, MESSENGER, and the Hubble Space Telescope, Astronomical Journal, Volume 158, Number 126, 2019.

[23] S. Blanco-Cuaresma, C. Soubiran, P. Jofre, U. Heiter, The Gaia FGK benchmark stars, Astronomy \& Astrophysics, Volume 566, A98, 2014.

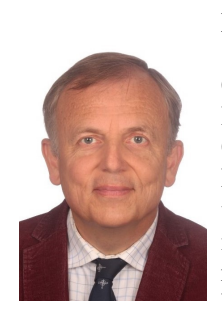

Martin J. Burgdorf received the Ph.D. degree in 1990 from Ruprecht-Karls-Universität Heidelberg, Germany.

$\mathrm{He}$ was a Systems Engineer at JenaOptronik/HE Space for METimage on EUMETSAT Polar System - Second Generation, where he was involved in preparing the calibration of this instrument. In 2015 he joined the working group on remote sensing of the Meteorological Institute of Universität Hamburg to become part of FIDUCEO (Fidelity and Uncertainty in Climate data records from Earth Observations), a HORIZON 2020 project. Since 2019 he works for MW-Luna, a project of Deutsche Forschungsgemeinschaft.

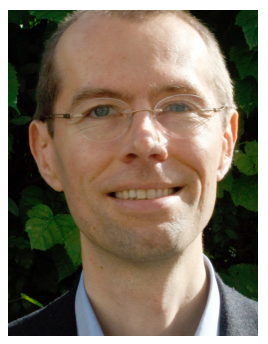

Stefan A. Buehler Stefan A. Buehler received his $\mathrm{PhD}$ in 1998 from the University of Bremen. He had a professorship at the University of Luleå (located in Kiruna, Sweden) between 2006 and 2013. Since 2013, he has a professorship at the University of Hamburg, where he currently serves as director of the Meteorological Institute. His main research interests are radiative transfer (Atmospheric Radiative Transfer Simulator, ARTS), remote sensing (ESA missions Metop-SG/ICI and FORUM) and climate physics (in particular the radiation budget and the feedbacks by water vapor and clouds).

Viju John Viju O. John received the M.S. degree in Physics and the M.S. degree in Atmospheric Science from the Cochin University of Science and Technology, Kochi, India, in 1998 and 2000, respectively, and the Ph.D. degree in physics from the University of Bremen, Bremen, Germany, in 2005. He is currently working as a Climate product Expert with the European Organisation for the Exploitation of Meteorological Satellites (EUMETSAT), Darmstadt, Germany. His research interests include the remote sensing of the Earth's atmosphere, inter-calibration of satellite sensors, evaluation climate models using satellite observations, and generation of climate data records of essential climate variables tailored for climate monitoring and for assimilation in climate reanalyses.

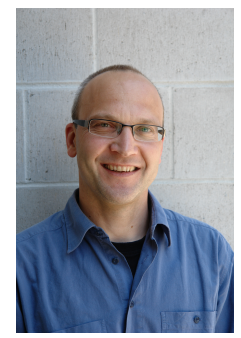

Thomas G. Müller Thomas G. Müller received his Ph.D. degree in 1997 from Ruprecht-KarlsUniversität Heidelberg, Germany. He was member of several infrared space projects (working for ESA and two Max-Planck-Institutes), and deeply involved in infrared instrument calibration activities. He has developed thermophysical model solutions for asteroids, planets and moons which are widely used as photometric and spectroscopic celestial calibrators in the thermal infrared, submillimetre and millimeter regime, for ground-, airborne and space projects Since 2002 he works at the Max-Planck-Institute for Extraterrestrial Physics in Garching, Germany. 